\section{Adipositas bei Rheuma: Einfluss auf Wirksamkeit von DMARDs untersucht}

\author{
Schäfer M et al. Obesity reduces the real-world \\ effectiveness of cytokine-targeted but not \\ cell-targeted disease-modifying agents in \\ rheumatoid arthritis. Rheumatology 2020; 59 : \\ 1916-1926
}

Rheumatologen aus Deutschland untersuchten den kombinierten Einfluss von Adipositas, Therapie und Geschlecht auf die Wirksamkeit häufig verwendeter DMARDs zur Therapie der rheumatoiden Arthritis (RA).

Ausgewertet wurden die Daten von Patientinnen und Patienten mit RA der deutschen Observationsstudie RABBIT (Rheumatoid Arthritis: Observation of Blologic Therapy) seit dem Jahr 2009. Eingeschlossen wurden Patientinnen und Patienten mit einem BMI $>18,5 \mathrm{~kg} / \mathrm{m}^{2}$, mindestens einer Nachuntersuchung und der Möglichkeit einer Nachbeobachtungszeit von mindestens einem halben Jahr.

Die Therapien wurden nach ihrem Wirkmechanismus gruppiert. Es wurden 5 Arzneimittel oder Gruppen von Arzneimitteln in Betracht gezogen: csDMARDs (z. B. MTX, SSZ, LEF und Chloroquin), TNF- Inhibitoren (Adalimumab, Certolizumab, Etanercept, Golimumab und Infliximab [INF]), Abatacept (ABA), Rituximab (RTX) und Tocilizumab (TOC). Für Etanercept, INF und RTX wurden sowohl das Originalarzneimittel als auch die verfügbaren Biosimilars (SB4, GP2015, CT-P13, SB2 und GP2013) berücksichtigt. Für die anderen bDMARDs wurden den Patienten nur Originalpräparate verschrieben. Als Maß für die Wirksamkeit galten eine Verbesserung des DAS28-ESR sowie seiner Komponenten während der ersten 6 Monate der Behandlung.

Die Studienpopulation bestand aus 10593 Patientinnen und Patienten. Davon waren 7845 Frauen, darunter 2192 übergewichtig, und 2748 Männer, darunter 718 übergewichtig. Zu Studienbeginn waren Alter und Geschlechtsverteilung zwischen adipösen und nicht adipösen Patientinnen und Patienten vergleichbar. Die übergewichtigen waren weniger seropositiv und hatten weniger erosive Gelenkveränderungen als die nicht übergewichtigen Studienteilnehmer. Sie hatten jedoch häufiger 3 oder mehr Komorbiditäten.

Für Frauen, die mit TNF-Inhibitoren oder csDMARDs behandelt wurden, sowie bei allen Patientinnen und Patienten, die mit TOC behandelt wurden, hatte eine Adipositas nach 6-monatiger Behandlung einen negativen Einfluss auf die Verbesserung der DAS28-ESR.

Die geringere DAS28-ESR-Verbesserung bei adipösen Frauen schien hauptsächlich mit einer schlechteren ESR/Entzündungsreaktion in der csDMARD-Gruppe assoziiert zu sein, während sie in der TNF-InhibitorenGruppe zusätzlich mit einer schwächeren Reaktion hinsichtlich Gelenkschmerzen sowie des allgemeinen Gesundheitsempfinden bei diesen Patientinnen assoziiert schien.

Für diejenigen, die mit TOC behandelt wurden, zeigten sich signifikante Assoziationen zwischen Adipositas und Gelenkschmerzen, nur bei Frauen. Nur bei Männern fanden signifikante Assoziationen zwischen Adipositas und einer schlechteren ESR-Antwort. Adipositas hatte hingegen keine negativen Auswirkungen auf die Wirksamkeit einer Therapie mit Rituximab und Abatacept.

\section{FAZIT}

Adipositas wirkt sich negativ auf die Wirksamkeit einer Zytokin-gerichteten Therapie, aber nicht auf eine Zell-gerichtete Therapie aus und beeinflusst mehr Ergebnis und Behandlung bei Frauen als bei Männern. Für Rituximab und Abatacept wurden hingegen keine Auswirkungen einer Adipositas auf die Wirksamkeit der Behandlung festgestellt, fassen die Autorinnen und Autoren ihre Ergebnisse zusammen.

Richard Kessing, Zeiskam 